\title{
Quantum Key Distribution Using Three Basis States
}

\author{
Subhash Kak \\ Department of Electrical \& Computer Engineering \\ Louisiana State University \\ Baton Rouge, LA 70803-5901; kak@ee.1su.edu
}

September 17, 2018

\begin{abstract}
This note presents a method of public key distribution using quantum communication of $n$ photons that simultaneously provides a high probability that the bits have not been tampered. It is a variant of the quantum method of Bennett and Brassard (BB84) where the transmission states have been decreased from 4 to 3 and the detector states have been increased from 2 to 3 . Under certain assumptions regarding method of attack, it provides superior performance (in terms of the number of usable key bits) for $n<18 m$, where $m$ is the number of key bits used to verify the integrity of the process in the BB84-protocol.

Keywords: Quantum cryptography, Quantum key distribution PACS Nos: $03.65 \mathrm{Bz}$
\end{abstract}

\section{Introduction}

Since the act of measurement reduces the state function of a quantum object, one cannot determine what state characterized the system prior to the measurement. This property is at the heart of the method of quantum key distribution which, under certain assumptions, provides an absolutely secure method of sharing a secret subject to public exchange of side information. As an example of quantum communication, the problem of key distribution forms a part of the general subject of quantum information science that has relevance for our understanding of the foundations of quantum and information theories.

In cryptography, two users, by convention called Alice (A) and Bob (B), wish to exchange information which must remain inaccessible to Eve (the eavesdropper). The quantum key distribution method of Bennett and Brassard (1984) (BB84), presented first at a conference in Bangalore sixteen years ago, uses 4 different polarizations of the photons and a pair of basis states in the detector 
for this information exchange. The BB84 protocol is symmetric in its use of the polarizations. After the key has been obtained, this protocol requires the exchange of further information about parity of randomly chosen subsets of the key.

Can we devise a scheme, somewhat in the spirit of joint encryption and error-correction coding (Kak 1985), that will not only distribute the key but also provide additional information about the integrity of the distribution process? In particular, we would like to have a method where the additional exchange of information, that is required after the key has been distributed, is unnecessary.

Given the symmetry of the BB84 method, one would expect that it could not be improved upon. But symmetry-breaking can provide advantage for part of the range of the operation of the system, albeit at the cost of some leakage of information. In this note we consider a variant protocol that breaks the symmetry of the BB84 method. In our method, three polarization states of the photons and three basis states of the detector are used. We show that doing so provides advantage over the BB84 method for certain sizes of the key under certain, not unreasonable, assumptions about the attacks mounted on it.

\section{The BB84 protocol}

In the BB84 method Alice (A) chooses photons (or other particles) prepared in four polarization states of $0,45,90$ and 135 degrees and sends $n$ of them in random order to Bob (B), who measures each photon using detectors matched in a random sequence to two pairs of orthogonal bases: $(0,90)$ and $(45,135)$ degrees. Note that if the directions and the polarizations of $0,45,90$ and 135 degrees are represented by $0, k, 1$ and $l$, respectively, it is sufficient to have the directions 0 and $k$ as the bases in the detector. If it is assumed that the photons are sent according to a clock, when the detector outputs nothing $(e)$, it is clear that the input was a photon in a state orthogonal to the detector setting.

Bob now tells Alice the sequence of the bases he used for his detection. Alice informs him which detector bases were correctly aligned. Alice and Bob keep only the data from these correctly measured (or inferred) photons, discarding the rest. Now Alice and Bob test their key by publicly choosing a random subset of bit positions and verifying that this subset has the same parity (defined as odd) in their respective versions of the key. If their keys had differed in one or more bit positions, this test would have discovered that fact with probability of $\frac{1}{2}$. One bit is now discarded, to compensate for the information leaked when its parity was revealed. This step is repeated $m$ times, leading to a certification with probability of $1-2^{-m}$ that their mutual keys are identical, at the cost of reducing the key by $m$ bits. Since the average number of correct alignments between the input bases and the detection pairs is about half of the total number of photons sent, the expected size of the key, certified with the probability $1-2^{-m}$, is $\frac{n}{2}-m$. 
This protocol assumes that Eve, the eavesdropper, cannot make multiple copies of the transmitted photons, perform various tests on them, and estimate their original polarizations using the public channel information from Alice. Should Eve intercept the photons directly, make measurements and transmit the measured photons to Bob, it would change the polarizations and this would be detected by Alice and Bob in their exchanges of the parity of the random subsets of the exchanged key.

It is interesting that there is a certain complementarity in the workings of classical and quantum communication. The state of a classical object can be fully determined and duplicated leading to ease of storage and transmission, and difficulty of secrecy-coding. On the other hand, the state of the quantum object is not fully known, so it is hard to duplicate it and hard to store and transmit it, while secrecy-coding is easy. The information associated with classical and quantum processing is also different (Kak 1998).

\section{$3 \quad$ Key distribution with three states}

In our asymmetric method, photons are prepared by Alice in the polarization states of of 0,45 and 90 degrees only. At the receiving end, Bob uses filters before his detector that are matched to the same polarizations. We have introduced asymmetry at two places: by cutting down on the number of photon polarizations from 4 to 3 , and by increasing the number of detector states from 2 (which is equivalent to 4 ) to 3 .

Table 1 summarizes the 9 different possibilities between the photon and the detector states for the data from Alice, Bob's detector settings, and what Bob actually receives.

Table 1: Photon and detector states

\begin{tabular}{||l|ccccccccc||}
\hline Alice's data & 0 & 0 & 0 & $\mathrm{k}$ & $\mathrm{k}$ & $\mathrm{k}$ & 1 & 1 & 1 \\
\hline Bob's filter & 0 & $\mathrm{k}$ & 1 & 0 & $\mathrm{k}$ & 1 & 0 & $\mathrm{k}$ & 1 \\
\hline Bob receives & 0 & $\mathrm{k} / \mathrm{e}$ & $\mathrm{e}$ & $0 / \mathrm{e}$ & $\mathrm{k}$ & $1 / \mathrm{e}$ & $\mathrm{e}$ & $\mathrm{k} / \mathrm{e}$ & 1 \\
\hline
\end{tabular}

Bob sends information about his filter settings to Alice on a public channel, and Alice uses the same public channel to tell Bob which settings were correct. The latter information makes it possible for Bob to infer which $e$ outputs should be replaced by either a 0 or 1 . For example, if Bob is told by Alice that his setting " 1 " is correct in the 3rd column of the Table, he would know that he should replace his estimate of the transmitted polarization from "nothing" $(e)$ to 0 .

As we can see, out of these 9 cases Bob is able to correctly receive or infer 5 cases. Now Eve, the eavesdropper, being unable to intercept or duplicate the photons being sent by Alice to Bob, can only work on the information being exchanged on the public channel. The only case where she would know the 
correct output is when Bob's filter setting is $\mathrm{k}$ and it is declared correct by Alice. This means that she knows one of the 5 correct cases of Bob. So while the correct recognition rate for Bob is $\frac{5}{9}$, bits at a rate of only $\frac{4}{9}$ have guaranteed security. Just these 4 bits out of the 9 listed in the Table will actually be used for creating the key, the fifth bit (the setting of $\mathrm{k}$ ) helps in authenticating the integrity of the process. The latter bits, at the rate of $\frac{1}{9}$, provide confirmation that there has been no tampering with the transmitted photons.

There is a $1 / 3$ probability that Eve would have used the correct filter placement at that spot if she had intercepted the photon sequence and replaced it with her own, so the transmission for every 9 bits can be certified to the probability $1-1 / 3=2 / 3$.

Quantum information is properly examined only in terms of the arrangement of the system and the experimenter. We assume here that Eve, like Bob, is using a receiver with settings of 3 basis states with the specified directional information. The certification probabilities quoted in this paper are based on this assumption. Unlike the BB84-method, Eve can, by using filter settings stuck at 0 or 1 , know the polarization of one-third of the transmitted photons. But that way, she could never hope to guess all the polarization choices made by Alice, and so this would not constitute a practical method of attack. To understand this clearly, assume that Eve's filter settings are all at 0 . She would receive a transmitted 0 as 0 , a transmitted 1 as e, and a transmitted $\mathrm{k}$ as 0 or e. One half of her received bits are 0 and the other half are 1 , and she is unable to work backwards and guess any specific value from her data alone.

\section{A probabilistic analysis}

Prior to being told the correct locations, the average mutual information, $I(A ; B)$, between Alice and Bob is computed by

$$
I(A ; B)=H(A)+H(B)-H(A, B) .
$$

where $H(A), H(B)$, and $H(A, B)$ are the individual entropy measures of $A, B$, and the joint entropy of $A$ and $B$, respectively.

$H(A)$ equals $\log 3 \approx 1.585$ bits. $H(B)$ is computed by first finding the probabilities of the four received states, $0, k, 1, e$, which are easily seen to be $1 / 6,2 / 9,1 / 6,4 / 9$, respectively. From this we find that

$$
H(B)=15 / 9 \log 3-7 / 9 \approx 1.864 \text { bits } .
$$

Likewise, the joint entropy, $H(A, B)$, is easily computed from the table of probabilities.

$$
H(A, B)=15 / 9 \log 3+5 / 9 \approx 3.197 \text { bits. }
$$

In other words, the amount of information leaking in this system is $I(A ; B)=$ $\log 3-4 / 3 \approx 0.252$ bits/photon, or about $16 \%$. Or the actual uncertainty from the point of Bob or Eve is $\log 3-0.252=4 / 3$ bits per photon. 
Since the k-photons (that constitute 1 out of every 3 photons transmitted) do not ultimately contribute to the formation of the key, the information being sent out by Alice is at the rate of $2 / 3 \times 4 / 3=8 / 9$ bits. With a $50 \%$ uncertainty for each photon, only half of them wil lead to the key sequence, so the information rate is now $1 / 2 \times 8 / 9=4 / 9$ bits, which is precisely the value we have argued using a different reasoning. This analysis confirms the information exchange figures associated with our protocol.

\section{When $\mathrm{n}$ photons are transmitted}

When $\mathrm{n}$ photons are transmitted we will, on an average, be able to obtain $\frac{4 n}{9}$ bits for the key and an additional $\frac{n}{9}$ bits for authentication of the transmission process. In other words, the transmission would be certified to the probability $1-3^{-n / 9}$.

In comparison, the 4 -state BB84 protocol provides $\frac{n}{2}-m$ key bits and certification of $1-2^{-m}$. When $n / 2-m=4 n / 9$, the key bits are the same number for the two cases, or for $n=18 \mathrm{~m}$.

We obtain more key bits from the 3-state protocol compared to the BB84protocol for $n<18 m$. Thus for $n=54$ and $m=6$, the 3 -state protocol gives 24 usable bits, while the BB84-protocol gives only 21 usable bits.

Furthermore, the certification probability is higher. The two certification probabilities will be the same if

$$
1-3^{-n / 9}=1-2^{-m} .
$$

Or BB84-certification probability is higher for $m>0.176 n$. But this is an impossible range of comparison because the effective value of $m$ in the 3 -state protocol is only $n / 9$.

We stress again that the certification probability comparisons are only notional, based on our assumption of a 3-state detection scheme used by Eve. Real comparison would require that the nature of the attack strategy be spelt out.

\section{Concluding remarks}

By weakening some assumptions regarding the nature of the quantum cryptographic system, we have devised a new 3-state protocol that has some attractive features, and it provides unexpectedly good results. It has the unique property that no further exchange of verification information is necessary after the initial steps of the protocol, unless one desires the certification probability to be greater than what is inherent in the system.

Quantum key distribution systems described here and elsewhere (Bennett 1992; Ekert 1991) are fundamentally dual in nature in as much that part of the information must be sent over a classical channel, as happens in the post-photon 
transmission communications between Alice and Bob. This aspect makes the communication system somewhat similar to those brain models that postulate an underlying quantum basis to cognitive processes (e.g. Hameroff 1998; Kak 1996). Is the "understanding" of the incoming sensory stimuli facilitated by the filtering information mapped into the neural structures of the brain during the process of evolution? And if the universe of the stimuli is itself structured, as is the case for the polarizations chosen by Alice, then is the subject led to an understanding of this structure by trying out various "stuck" settings of his receiver apparatus and exploiting the fact that biological signals are often repetitive?

\section{References}

Bennett, C H and Brassard, G 1984 "Quantum cryptography: Public key distribution and coin tossing," Proceedings of the IEEE Intl. Conf. on Computers, Systems, and Signal Processing, Bangalore, India (IEEE New York, 1984, pages 175-179).

Bennett, C H 1992 Phys. Rev. Lett. 68, 3121

Ekert, A K 1991 Phys. Rev. Lett. 67, 661

Hameroff, S 1998 Phil. Trans. R. Soc. Lond. A 356, 1869

Kak, S 1985 IEEE Trans. on Computers C-34, 803

Kak, S 1996 "The three languages of the brain: Quantum, reorganizational, and associative." In Learning as Self-Organization, K. Pribram and J. King (eds.). (Lawrence Erlbaum Associates, Mahwah, 1996, pages 185219).

Kak, S 1998 Foundations of Physics 28, 1005 